\title{
Choices of Expansion and Testing Functions for the Method of Moments Applied to a Class of Electromagnetic Problems
}

\author{
M. I. Aksun, Member, IEEE, and Raj Mittra, Fellow, IEEE
}

\begin{abstract}
It is well known that the choice of expansion and testing functions plays an important role in determining the rate of convergence of the integrals associated with the moment method matrix, and that an improper choice can lead to erroneous results. The main objective of this paper is to critically examine this convergence issue and to provide criteria for the choice of these expansion and testing functions. The question of whether these functions need to satisfy the Holder condition is also investigated and the convergence behavior of the integrals involved in the spatial and spectral domain moment method is discussed for some representative expansion and testing functions.
\end{abstract}

\section{INTRODUCTION}

$\mathbf{T}$ THE METHOD of moments (MoM) [1], which is one of the most commonly used numerical techniques for solving electromagnetic problems, is based upon the transformation of an operator equation into a matrix equation. While the computation of the matrix elements in the MoM can be carried out relatively efficiently when the medium involved is free-space, the introduction of a substrate material backed by a ground plane can render this task extremely time-consuming because of the need to compute the Sommerfeld's integrals appearing in the Green's functions. One approach to circumventing this difficulty is to work in the spectral domain [2] where the closed-form expressions for the Green's functions can be obtained in a relatively straightforward manner [3]. Another solution to this problem has been recently developed [4]-[6] in the spatial domain where the Green's functions corresponding vector and scalar potentials were cast into closedforms. Both the spatial and spectral domain approaches have been successfully applied to many electromagnetic problems involving microstrip-type structures. However, the questions pertaining to the choice of the expansion and testing functions in formulating the MoM matrix have not been addressed in much detail except in the context of linear spaces and operators strictly from a mathematical point of view [7], [8], and briefly in connection with the spectral Galerkin's

Manuscript received May 1, 1992; revised July 20, 1992

M. I. Aksun was with the Electromagnetic Communication Laboratory, Department of Electrical and Computer Engineering, University of Illinois at Urbana-Champaign, 1406 W. Green St., Urbana, IL 61801. He is presently with the Department of Electrical and Electronics Engineering, Bilkent University, Bilkent, Ankara 06533, Turkey.

R. Mittra is with the Electromagnetic Communication Laboratory, Department of Electrical and Computer Engineering, University of Illinois at Urbana-Champaign, 1406 W. Green St., Urbana, IL 61801.

IEEE Log Number 9205455. method [9]; consequently, there are still some open questions regarding the use of certain expansion and testing functions for the operator equations that are used in electromagnetic problems [10]-[13]. The purpose of this paper is to show mathematically that there is an admissible class of functions for expansion (testing) functions depending upon the choice of testing (expansion) functions. If one choose these functions such that they fall outside of the admissible class, the integrals involved in the application of the MoM can be divergent and the results can be erroneous. Additionally, the issues involving the slow convergence of the integrals often encountered in the spectral domain, and the handling of discontinuous expansion functions at junctions or corners, and in the source excitation region, are also addressed.

We begin by discussing briefly, in Section II, the application of the moment method in the spatial and spectral domains and by deriving the expressions for the MoM matrix elements that turn out to be improper integrals. The convergence of these integrals is studied for different combinations of expansion and testing functions in both the spatial and spectral domains. This is followed in Section III, with a discussion on the convergence behavior of the integrals involved for a representative choice of expansion and testing functions.

\section{CONVERGENCE CRITERIA FOR THE INTEGRALS OF THE METHOD OF MOMENTS}

Consider, for the sake of illustration, a general microstrip structure shown in Fig. 1 where it is assumed that the substrate layer extends to infinity in the transverse directions. Let the thickness and the permittivity of the substrate be denoted by $d$ and $\epsilon_{r}$, respectively. Although the Green's functions discussed herein pertain to the geometry shown in Fig. 1, the comments appearing below apply to more general geometries as well.

\section{A. Convergence Study in the Spatial Domain}

Let us begin by representing the electric fields in terms of scalar and vector potentials, $\phi$ and $\boldsymbol{A}$, respectively,

$$
\boldsymbol{E}=-j \omega \boldsymbol{A}-\nabla \phi
$$

The vector and scalar potentials can, in turn, be represented in terms of convolution integrals, involving the surface current density $J$ on the patch, as

$$
\boldsymbol{A}=\overline{\boldsymbol{G}}^{A} * \boldsymbol{J}
$$




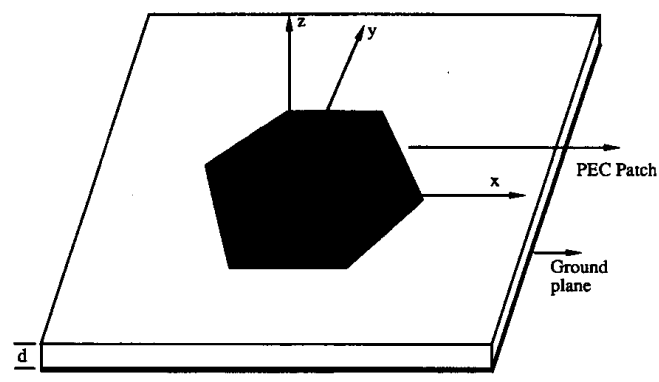

Fig. 1. A general microstrip structure.

$$
\phi=G_{q} *\left(\frac{-1}{j \omega} \nabla \cdot J\right)
$$

where $G^{A}$ is the dyadic Green's function of the vector potential and $G_{q}$ is the Green's function of the scalar potential. From (2), the tangential electric fields on the plane of the patch $(z=0)$ can be written as

$$
\begin{aligned}
E_{x}= & -j \omega G_{x x}^{A} * J_{x} \\
& +\frac{1}{j \omega} \frac{\partial}{\partial x}\left[G_{q} * \nabla \cdot \boldsymbol{J}\right] \\
E_{y}= & -j \omega G_{y y}^{A} * J_{y} \\
& +\frac{1}{j \omega} \frac{\partial}{\partial y}\left[G_{q} * \nabla \cdot \boldsymbol{J}\right]
\end{aligned}
$$

It may be noted that the spatial domain Green's functions, appearing in (3), have algebraic singularities of the first order; that is, $G \sim O\left(1 /\left|\boldsymbol{r}-\boldsymbol{r}^{\prime}\right|\right)$ as $\boldsymbol{r} \rightarrow \boldsymbol{r}^{\prime}$, where $\boldsymbol{r}$ and $\boldsymbol{r}^{\prime}$ are the observation and source coordinates, respectively.

To solve for the surface current density on the patch by the MoM, the first step is to expand the surface current densities by a linear combination of the expansion functions as follows:

$$
\begin{aligned}
& J_{x}=\sum_{n} A_{n} J_{x n}(x, y)+J_{x s}(x, y) \\
& J_{y}=\sum_{n} B_{n} J_{y n}(x, y)+J_{y s}(x, y)
\end{aligned}
$$

where $A_{n}$ and $B_{n}$ are the unknown coefficients of the expansion functions $J_{x n}$ and $J_{y n}$, and $J_{x s}$ and $J_{y s}$ are the source representations. These expansion functions can be subsectional, traveling-wave, or entire-domain type depending upon the geometry under consideration. Next we substitute (4) into (3) and test the resulting equations using the functions $T_{x m}$ and $T_{y m}$ and a suitable definition of inner product, e.g.,

$$
\langle f, g\rangle=\iint d x d y f^{*}(x, y) g(x, y)
$$

The process of testing could be interpreted as satisfying the boundary conditions on the tangential electric fields on the patch in the integral sense, provided that the domain of the testing functions covers the entire patch. Since the testing functions and the tangential electric fields are finite on the complementary regions, the left-hand sides of (3a) and ( $3 b)$ become zero after the testing, and the following algebraic equations for the coefficients $A_{n}$ and $B_{n}$ are obtained for each $m$ in (6a) and (6b) at the bottom of the page, where $\boldsymbol{J}_{s}=J_{x s} \boldsymbol{x}+J_{y s} \boldsymbol{y}$.

Note that some of the inner products in (6) contain differentiations that result in higher order singularities in the corresponding integrands than those that do not contain these derivatives. Thus, it will be sufficient to study only the terms with the derivatives in order to ensure the convergence of all the integrals. In general, each inner product term in the spatial domain is a five-dimensional integral: one of these is associated with the Green's function itself; two of them are convolution integrals; and, the other two are the inner products defined in (5). Since the numerical integration of the five-dimensional integrals is very expensive, the convolution over the Green's function and the expansion function is often transferred over to the expansion and testing functions and the convolution is performed analytically. This transfer of the convolution requires an interchange of the order of the integrals, which is possible if the original integral is uniformly convergent. Here, we will use the following sufficiency criterion [14] for the uniform convergence of the integral:

$$
\left|F\left(x_{1}, x_{2}, \cdots, x_{n} ; x_{1}^{\prime}, x_{2}^{\prime}, \cdots, x_{n}^{\prime}\right)\right|<\frac{A}{r^{n-\alpha}}
$$

where $F\left(x_{1}, x_{2}, \cdots, x_{n} ; x_{1}^{\prime}, x_{2}^{\prime}, \cdots, x_{n}^{\prime}\right)$ is the integrand given in a bounded domain; $A$ is a constant; $\alpha>0$; and $r$ denotes the distance between $\left(x_{1}, x_{2}, \cdots, x_{n}\right)$ and $\left(x_{1}^{\prime}, x_{2}^{\prime}, \cdots, x_{n}^{\prime}\right)$.

The issue regarding the choice of the expansion and testing functions can now be discussed by investigating the elements of (6a) and (6b). Since the strongest conditions on the expansion and testing functions for the convergence of the integrals

$$
\begin{aligned}
& \sum_{n} A_{n}\left\{\left\langle T_{x m}, G_{x x}^{A} * J_{x n}\right\rangle+\frac{1}{\omega^{2}}\left\langle T_{x m}, \frac{\partial}{\partial x}\left[G_{q} * \frac{\partial}{\partial x} J_{x n}\right]\right\rangle\right\}+\sum_{n} B_{n}\left\{\frac{1}{\omega^{2}}\left\langle T_{x m}, \frac{\partial}{\partial x}\left[G_{q} * \frac{\partial}{\partial y} J_{y n}\right]\right\rangle\right\} \\
& =-\left\langle T_{x m}, G_{x x}^{A} * J_{x s}\right\rangle-\frac{1}{\omega^{2}}\left\langle T_{x m}, \frac{\partial}{\partial x}\left[G_{q} * \nabla \cdot J_{s}\right]\right\rangle \\
& \sum_{n} B_{n}\left\{\left\langle T_{y m}, G_{y y}^{A} * J_{y n}\right\rangle+\frac{1}{\omega^{2}}\left\langle T_{y m}, \frac{\partial}{\partial y}\left[G_{q} * \frac{\partial}{\partial y} J_{y n}\right]\right\rangle\right\}+\sum_{n} A_{n}\left\{\frac{1}{\omega^{2}}\left\langle T_{y m}, \frac{\partial}{\partial y}\left[G_{q} * \frac{\partial}{\partial x} J_{x n}\right]\right\rangle\right\} \\
& =-\left\langle T_{y m}, G_{y y}^{A} * J_{y s}\right\rangle-\frac{1}{\omega^{2}}\left\langle T_{y m}, \frac{\partial}{\partial y}\left[G_{q} * \nabla \cdot J_{s}\right]\right\rangle
\end{aligned}
$$


would be set by the term containing the double derivatives with respect to the same variable, only the integral containing $J_{x n}$ and $T_{x m}$ will be studied in detail. This integral is:

$$
\begin{aligned}
\left\langle T_{x m},\right. & \left.\frac{\partial}{\partial x}\left[G_{q} * \frac{\partial}{\partial x} J_{x n}\right]\right\rangle \\
= & \iint_{D(T)} d x d y T_{x m}(x, y) \frac{\partial}{\partial x} \iint_{D(B)} d x^{\prime} d y^{\prime} \\
& \cdot G_{q}\left(x-x^{\prime}, y-y^{\prime}\right) \frac{\partial}{\partial x^{\prime}} J_{x n}\left(x^{\prime}, y^{\prime}\right)
\end{aligned}
$$

where $D(T)$ and $D(B)$ represent the domain of the testing and expansion functions, respectively, and the second term in the inner product is the tangential derivative of the scalar potential If one were to calculate the derivative of the inner double integral in (8), which is a convolution integral, the derivative of the expansion function in this integral has to satisfy the Holder condition [15]. This condition is somewhat stronger than the continuity condition but weaker than the differentiability condition [16]. However, in the MoM, we are interested in calculating the inner product of the derivative of the scalar potential with a testing function. If the testing function is at least piecewise continuous, we can use integration by parts to transfer the differentiation of the scalar potential over to the testing function as follows

$$
\begin{aligned}
\left\langle T_{x m}\right. & \left.\left., \frac{\partial}{\partial x}\left[G_{q} * \frac{\partial}{\partial x} J_{x n}\right]\right]\right\rangle \\
= & \left.\int d y T_{x m}(x, y)\left(G_{q} * \frac{\partial}{\partial x} J_{x n}\right)\right|_{x \in \Omega(T)} \\
& -\left\langle\frac{\partial}{\partial x} T_{x m}, G_{q} * \frac{\partial}{\partial x} J_{x n}\right\rangle
\end{aligned}
$$

where the expression in the braces is evaluated at the boundaries of the testing functions, denoted here by $\Omega(T)$. Once this is done, the restriction on the charge density to satisfy the Holder condition can be lifted. The step involving the integration by parts must be justified, because the piecewise differentiable expansion functions cannot ensure the existence of the tangential derivative of the scalar potential. In accordance with the discussion presented in [17], the existence in Riemann sense of either one of the integrals in (9) also implies the existence of the other. The integral in the right hand side of (9) is guaranteed to satisfy the Riemann's integrability condition if the testing functions are at least piecewise continuous functions in the direction of differentiation while the expansion functions are piecewise differentiable or smoother.

Similar arguments can be applied to the second inner product term in (6b) where the differentiations are with respect to $y$. Note also that the other terms which have derivatives along $\mathrm{x}$ as well as $\mathrm{y}$ directions do not require as strong a condition on the expansion and testing functions as do the terms that contain both of these differentiations in the same direction. As a consequence, the expansion functions for the current density should be chosen at least from a class of piecewise continuous functions and the testing functions should in turn be selected from piecewise differentiable functions, or vice versa. These conditions apply to these functions along the differentiation of the scalar potentials. This, in turn, implies that

$$
\begin{aligned}
J_{x n}(x, y) & \triangleq F_{x}(x) G_{x}(y) \\
J_{y n}(x, y) & \triangleq F_{y}(x) G_{y}(y) \\
T_{x n}(x, y) & \triangleq P_{x}(x) R_{x}(y) \\
T_{y n}(x, y) & \triangleq P_{y}(x) R_{y}(y)
\end{aligned}
$$

where $F_{x}(x), G_{y}(y) \in$ class of piecewise differentiable functions and $P_{x}(x), R_{y}(y) \in$ class of piecewise continuous functions, or vice versa. However, the functions can have square-root singularities in the direction orthogonal to that of the differentiation to model the singular behavior of the edge current, ie., $G_{x}(y), F_{y}(x), R_{x}(y)$ and $P_{y}(x) \in$ class of piecewise continuous functions with or without square-root singularities. The requirements on the testing and expansion functions can be relaxed by using higher order differentiable functions. For instance, if the chosen expansion (testing) functions are twice-differentiable, then piecewise continuous testing (expansion) functions or even impulse distributions are sufficient to ensure the convergence of the integral. Among the commonly used expansion and testing functions, piecewise sinusoids (PWS) and triangle functions are piecewise differentiable functions, pulse functions and any type of junction expansion functions, e.g., half triangle, half PWS, etc., are piecewise continuous functions.

It is essential to use piecewise continuous expansion functions, with finite discontinuities in the direction of the differentiation, for the geometries that have junctions, load or source connections in the domain of interest [18]-[20]. For these cases, the impulse functions generated by the differentiations of the expansion functions at the point of discontinuities should not be included in the calculations, because the divergence of the current in (3) must be finite at these junctions. This could be interpreted simply as requiring the continuity of the current at the junctions. This is tantamount to saying that the discontinuity of the current in one direction must equal to the negative of the discontinuity in the other direction such that their derivatives cancel each other at that point.

\section{B. Convergence Study in the Spectral Domain}

The tangential electric field on the plane of the patch due to the patch currents $J_{x}$ and $J_{y}$ can be written, in terms of the electric field Green's functions, as convolution type coupled integral equations as follows:

$$
\begin{aligned}
& E_{x}(x, y, z=0) \\
& \quad=\iint_{D(B)} d x^{\prime} d y^{\prime}\left[Z_{x x}\left(x-x^{\prime}, y-y^{\prime}\right) J_{x}\left(x^{\prime}, y^{\prime}\right)\right. \\
& \left.\quad+Z_{x y}\left(x-x^{\prime}, y-y^{\prime}\right) J_{y}\left(x^{\prime}, y^{\prime}\right)\right] \\
& E_{y}(x, y, z=0)
\end{aligned}
$$




$$
\begin{aligned}
= & \iint_{D(B)} d x^{\prime} d y^{\prime}\left[Z_{y x}\left(x-x^{\prime}, y-y^{\prime}\right) J_{x}\left(x^{\prime}, y^{\prime}\right)\right. \\
& \left.+Z_{y y}\left(x-x^{\prime}, y-y^{\prime}\right) J_{y}\left(x^{\prime}, y^{\prime}\right)\right]
\end{aligned}
$$

where the Green's function $Z_{i j}$ is the contribution of a unit j-directed electric current element at the microstrip to the electric field $E_{i}$ at the microstrip plane. In the spectral domain formulation, these equations are Fourier transformed to yield the following algebraic equations

$$
\begin{aligned}
\tilde{E}_{x}\left(k_{x}, k_{y}\right)= & \tilde{Z}_{x x}\left(k_{x} k_{y}\right) \tilde{J}_{x}\left(k_{x}, k_{y}\right) \\
& +\tilde{Z}_{x y}\left(k_{x}, k_{y}\right) \tilde{J}_{y}\left(k_{x}, k_{y}\right) \\
\tilde{E}_{y}\left(k_{x}, k_{y}\right)= & \tilde{Z}_{y x}\left(k_{x} k_{y}\right) \tilde{J}_{x}\left(k_{x}, k_{y}\right) \\
& +\tilde{Z}_{y y}\left(k_{x}, k_{y}\right) \tilde{J}_{y}\left(k_{x}, k_{y}\right)
\end{aligned}
$$

The electric field Green's functions in the spectral domain can be readily obtained by using the immittance approach described by Itoh [3]. The application of the moment method starts with the expansion of the current densities as in (4), substitution of the Fourier transforms of these expansion functions in (12) followed by the testing with the Fourier transforms of the testing functions. Following this procedure one arrives at the following algebraic equations:

$$
\begin{aligned}
& \sum_{n} A_{n}\left\langle\tilde{T}_{x m}, \tilde{Z}_{x x} \tilde{J}_{x n}\right\rangle \\
&+ \sum_{n} B_{n}\left\langle\tilde{T}_{x m}, \tilde{Z}_{x y} \tilde{J}_{y n}\right\rangle=0 \\
& \sum_{n} A_{n}\left\langle\tilde{T}_{y m}, \tilde{Z}_{y x} \tilde{J}_{x n}\right\rangle \\
&+\sum_{n} B_{n}\left\langle\tilde{T}_{y m}, \tilde{Z}_{y y} \tilde{J}_{y n}\right\rangle=0
\end{aligned}
$$

where the inner products are defined over an infinite domain. The convergence of the integrals is guaranteed provided that

$$
\left|F\left(k_{1}, k_{2}, \cdots, k_{n}\right)\right| \leq A k^{-n-\epsilon}
$$

where $F\left(k_{1}, k_{2}, \cdots, k_{n}\right)$ is the integrand, $k=\sqrt{k_{1}^{2}+k_{2}^{2}+\cdots+k_{n}^{2}}$ and $\epsilon>0$.

A knowledge of the leading terms of the asymptotic expansions of the Green's functions, expansion functions and testing functions is needed for establishing the convergence of the inner products in (13). The leading terms of the Green's functions are obtained from (A1)-(A6) in Appendix A and are given by

$$
\begin{aligned}
& \tilde{Z}_{x x} \rightarrow \frac{1}{j \omega \epsilon_{0}\left(1+\epsilon_{r}\right)} \frac{k_{x}^{2}}{\sqrt{k_{x}^{2}+k_{y}^{2}}} \\
& \tilde{Z}_{x y}=\tilde{Z}_{y z} \rightarrow \frac{1}{j \omega \epsilon_{0}\left(1+\epsilon_{r}\right)} \frac{k_{x} k_{y}}{\sqrt{k_{x}^{2}+k_{y}^{2}}} \\
& \tilde{Z}_{y y} \rightarrow \frac{1}{j \omega \epsilon_{0}\left(1+\epsilon_{r}\right)} \frac{k_{y}^{2}}{\sqrt{k_{x}^{2}+k_{y}^{2}}}
\end{aligned}
$$

As for the expansion and testing functions, it can be shown that they have the general behavior of the form;

$$
\begin{aligned}
\tilde{T}_{x m}\left(k_{x}, k_{y}\right) & \rightarrow O\left(\frac{1}{k_{x}^{\alpha} k_{y}^{\beta}}\right) ; \\
\tilde{J}_{x n}\left(k_{x}, k_{y}\right) & \rightarrow O\left(\frac{1}{k_{x}^{\gamma} k_{y}^{\eta}}\right) \\
\tilde{T}_{y m}\left(k_{x}, k_{y}\right) & \rightarrow O\left(\frac{1}{k_{x}^{\iota} k_{y}^{\kappa}}\right) ; \\
\tilde{J}_{y n}\left(k_{x}, k_{y}\right) & \rightarrow O\left(\frac{1}{k_{x}^{\nu} k_{y}^{\tau}}\right)
\end{aligned}
$$

The convergence of the inner products in (13) can now be investigated with a view to defining the minimum of the orders of $k_{x}$ and $k_{y}$. Note that the leading terms of the expansion and testing functions for large spectral variables have been chosen for the worst case conditions because their numerators have been assumed to be constant. We observe, for instance, that the Fourier transform of a pulse function is $\sin (k) / k$ whose integral over an infinite domain is convergent whereas that of $1 / k$ is divergent. Returning to the inner products in (13), the convergence of the integrals are investigated as follows:

i)

$$
\begin{aligned}
&\left\langle\tilde{T}_{x m}, \tilde{Z}_{x x} \tilde{J}_{x n}\right\rangle \rightarrow \iint_{-\infty}^{+\infty} d k_{x} d k_{y} \\
& O\left(\frac{1}{k_{x}^{\alpha} k_{y}^{\beta}}\right) \frac{k_{x}^{2}}{\sqrt{k_{x}^{2}+k_{y}^{2}}} O\left(\frac{1}{k_{x}^{\gamma} k_{y}^{\eta}}\right)
\end{aligned}
$$

The convergence of the above integral is guaranteed if one chooses $\alpha+\gamma>3$ and $\beta+\eta>1$. Some comments on the class of expansion and testing functions which satisfy the above criteria are given later in this section.

ii)

$$
\begin{aligned}
& \left\langle\tilde{T}_{x m}, \tilde{Z}_{x y} \tilde{J}_{y n}\right\rangle \rightarrow \iint_{-\infty}^{+\infty} d k_{x} d k_{y} \\
& O\left(\frac{1}{k_{x}^{\alpha} k_{y}^{\beta}}\right) \frac{k_{x} k_{y}}{\sqrt{k_{x}^{2}+k_{y}^{2}}} O\left(\frac{1}{k_{x}^{\nu} k_{y}^{\tau}}\right)
\end{aligned}
$$

For this integral, the conditions $\alpha+\nu>2$ and $\beta+\tau>2$ must be satisfied in order for the integral to converge.

Similarly, by considering the other two inner products in (13b), we can derive the conditions on the exponents of the spectral domain variables $k_{x}$ and $k_{y}$. These are: iii) $\iota+\gamma>2$ and $\kappa+\eta>2$, iv) $\iota+\nu>1$ and $\kappa+\tau>3$. The conditions given above are sufficient for the convergence of the integrals.

At this point it would be useful to list the asymptotic behaviors for large $k$ of a number of representative expansion and testing functions frequently employed in the moment method solutions to electromagnetic problems of the type being considered in this paper:

$\mathcal{F}\{\delta(x)\} \rightarrow O(1)$

$\mathcal{F}\{$ functions with square-root singularities $\} \leq O\left(J_{0}(k)\right)$ as $k \rightarrow \infty$ 
$\mathcal{F}$ \{piecewise continuous functions $\} \leq O(1 / k)$ as $k \rightarrow \infty$ $\mathcal{F}\{$ piecewise differentiable functions $\} \leq O\left(1 / k^{2}\right)$ as $k \rightarrow$ $\infty$

$\mathcal{F}$ \{piecewise twice-differentiable functions $\} \leq O\left(1 / k^{3}\right)$ as $k \rightarrow \infty$

where $\mathcal{F}$ denotes the Fourier transform and $k$ is the spectral domain variable. It should be emphasized that there exists the possibility that some of the functions in the same class might decay slightly faster than the others. As an example, we mention the triangular function, which is a piecewise differentiable function, and which has the Fourier transform in the form of $\sin ^{2}(k) / k^{2}$. As mentioned before, the worst case behaviors in the spectral domain are considered here so that the convergence for every function in a given class is assured.

For the specific geometry, one can refer to the convergence criteria and the classification of functions given above in order to facilitate the choice of the expansion and testing functions.

\section{RESUlTS AND DisCUSSIONS}

In this section we discuss several choices of the expansion and testing functions from the point of view of convergence. We draw from the pool of commonly used expansion and testing functions in electromagnetic problems, viz., the impulse, pulse, triangular, piecewise sinusoid, and entire domain functions. A number of different combination of expansion and testing functions are considered in the following:

(i) Both $J_{x}$ and $J_{y}$ represented by pulses in $\mathrm{x}$ as well as $\mathrm{y}$ directions: Since the pulse is a piecewise continuous function, it can not satisfy the Holder condition. The current expansion functions $J_{x n}$ and $J_{y n}$ are represented as $[P(x) \cdot P(y)]$, where $P(\cdot)$ denotes the pulse function. Both of the differentiations in the second inner product term in (6a) should be transferred to the testing function, implying that the testing function should be selected at least from the group of piecewise differentiable functions with respect to $x$. While the transfer of the differentiation on the expansion function may not be strictly valid in the classical sense, it is nonetheless legitimate to carry it out in the sense of distributions. From the examination of the third inner product in (6a), the testing function should at least be a piecewice continuous function in $\mathrm{y}$. In summary, the testing functions $T_{x m}$ and $T_{y m}$ could be triangular functions along their polarizations and pulse functions in the orthogonal directions of their polarizations. Although it might appear that this choice of the expansion and testing functions does not yield convergent integrals in the spectral domain the integrals are actually convergent albeit slowly. This is because the Fourier transforms of the pulse and triangular functions are proportional to $\operatorname{sinc}(k)$ and $\operatorname{sinc}^{2}(k)$, respectively, and these are less than or equal to $1 / k$ and $1 / k^{2}$ for $0 \leq k<\infty$, (sinc $\left.(k)<1 / k, \operatorname{sinc}^{2}(k) \leq 1 / k^{2}\right)$. The convergence of the integrals in the spectral domain can be improved by using the following expansion and testing functions. (ii) Both $J_{x}$ and $J_{y}$ represented by roof-tops: A roof-top consists of a triangular function in the direction of polarization and pulse function in the orthogonal direction to the polarization of the current. With this choice, the convergence is sufficiently rapid in the spectral domain and it is not necessary to transfer the inner

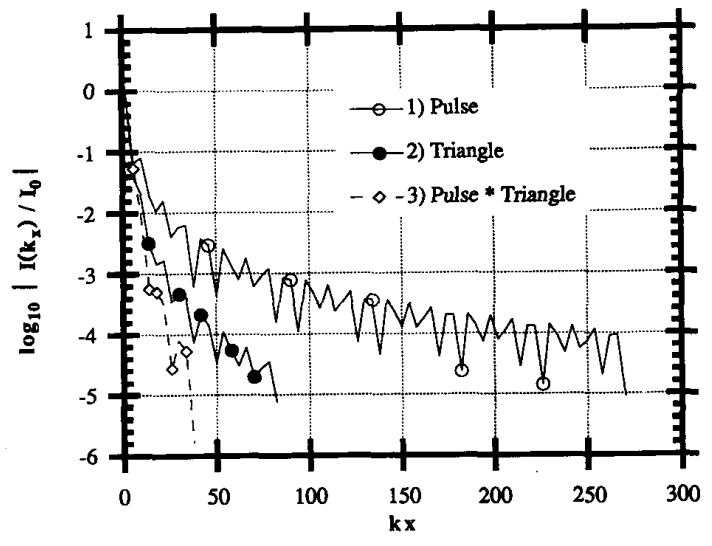

Fig. 2. Convergence of a spectral domain integral for a roof-top expansion function and three different testing functions; (1) [Pulse $(x)$ Pulse $(y)$ ]; (2) [Triangle $(x)$ Pulse $(y)]$; (3) [(pulse $(x) *$ Triangle $(x))$ Pulse $(y)]$. Vertical axis is $\log _{10}\left|I\left(k_{x}\right) / I_{0}\right|$ where $I$ is defined in (17) and $I_{0}=I\left(k_{x}\right)$ with $0<k_{x} \leq \Delta k_{x}$

differentiations to the testing functions in the spatial domain. There is, however, a disadvantage in using the higher order differentiable functions, namely their Fourier transforms are oscillatory. This could be seen by examining the pulse and triangular functions in the spectral domain. The use of the higher order differentiable functions as expansion or testing functions helps decrease the range of the integrations at the cost of increasing the complexity and computation time for the integrands. We will now illustrate the convergence of the following moment integral for three different choices for testing functions

$$
\begin{aligned}
I= & \int_{0}^{\infty} \int_{0}^{\infty} d k_{x} d k_{y} \tilde{T}_{x m}\left(k_{x}, k_{y}\right) \\
& \cdot \frac{k_{x}^{2}}{\sqrt{k_{x}^{2}+k_{y}^{2}}} \tilde{J}_{x n}\left(k_{x}, k_{y}\right)
\end{aligned}
$$

where

$$
\begin{aligned}
& \tilde{J}_{x n}\left(k_{x}, k_{y}\right)=\mathcal{F}\{\operatorname{Triangle}(x) \operatorname{Pulse}(y)\} \\
& =\operatorname{sinc}^{2}\left(k_{x} / 2\right) \operatorname{sinc}\left(k_{y}\right)
\end{aligned}
$$

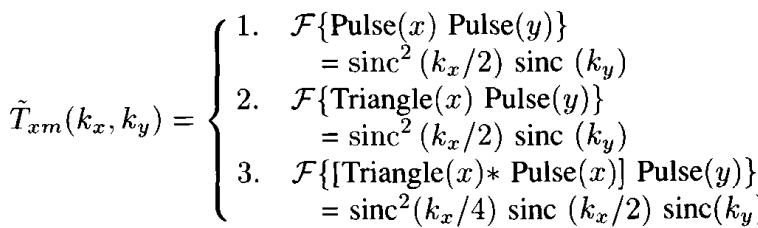

Fig. 2 shows the normalized contribution of integral I (17) on each sub-interval $\Delta k_{x}$ of $k_{x}$, until it becomes less than $10^{-5}$. We observe that the integration limit reduces significantly as one goes from the testing function 1 to 3 , viz., from $6.6 \mathrm{CPU}$ sec to $1.2 \mathrm{CPU}$ sec on the Cray Y/MP. (iii) An entire domain expansion function with a square-root singularity: Note that the square-root singularity appears not in the direction of the differentiations but in the orthogonal direction, (6). 
Consequently, the convolution integrals are still integrable because the order of singularity is less than 2 which is the criterion for the uniform convergence of the two-dimensional integrals (7). For this case, the differentiations in front of the convolution integrals are transferred over to the testing functions which might also have square-root singularities. Since the convolution integral of a Green's function and a function with a square-root singularity can yield a function which also has a square-root singularity, the total integrand can be a first-order singular function whose integral is convergent in a two-dimensional space. Hence, using a function which has a square-root singularity in the directions that are orthogonal of the direction of the differentiation as expansion and testing functions in (6) results in a convergent inner product.

It should also be noted that the point matching (impulse testing functions) can not be used unless the expansion functions are twice-differentiable in the variable of the impulse. The variation of the expansion function in the orthogonal direction should be chosen according to the variation of impulse in that direction, which could be decided by examining (5) in the spatial domain or given criteria in the spectral domain.

\section{CONCLUSIONS}

The choice of the expansion and testing functions is rather crucial in the MoM solution, because this choice plays an important role on the convergence of the integrals and, consequently, on the results. Through a thorough examination of the convergence question we conclude that the classes of functions from which the expansion and testing functions are chosen must satisfy the following criteria: (i) In the direction of the polarization of the current, the sum of the order of the differentiability of the expansion and testing functions must be equal to or greater than one; (ii) in the orthogonal direction of the polarization of the current, any piecewise continuous function or even functions with singularities of the order of less than one are admissible.

\section{APPENDIX A}

The Green's functions in (12) were obtained by using the spectral domain immittance approach [3] as

$$
\begin{aligned}
& \tilde{Z}_{x x}=\tilde{Z}^{e} \cos ^{2} k_{\theta}+\tilde{Z}^{h} \sin ^{2} k_{\theta} \\
& \tilde{Z}_{x y}=\left(\tilde{Z}^{e}-\tilde{Z}^{h}\right) \sin k_{\theta} \cos k_{\theta} \\
& \tilde{Z}_{y x}=\left(\tilde{Z}^{e}-\tilde{Z}^{h}\right) \sin k_{\theta} \cos k_{\theta} \\
& \tilde{Z}_{y y}=\tilde{Z}^{e} \sin ^{2} k_{\theta}+\tilde{Z}^{h} \cos ^{2} k_{\theta}
\end{aligned}
$$

where

$$
\begin{array}{rl}
\theta & =\tan ^{-1}\left(\frac{k_{y}}{k_{x}}\right) \\
\tilde{Z}^{h}=\frac{1}{Y_{i 0}+Y_{i 1} \operatorname{coth} \gamma_{1} d} i & i=\text { TM for } e \\
i=\text { Tor } h
\end{array}
$$

The TE and TM wave impedances are defined as

$$
Z_{\mathrm{TE} i}=\frac{j \omega \mu}{\gamma_{i}} \quad \text { and } \quad Z_{\mathrm{TM} i}=\frac{\gamma_{i}}{j \omega \epsilon_{i}} \quad \text { for } i=0,1
$$

and the complex wavenumbers are

$$
\gamma_{i}=\sqrt{k_{x}^{2}+k_{y}^{2}-k_{i}^{2}} \quad \text { for } i=0,1
$$

in the air and substrate, respectively.

\section{ACKNOWLEDGMENT}

Useful comments from Drs. E. Michielssen, S. Gedney and L. Gurel are gratefully acknowledged. Supercomputer time for this work was provided by the National Center for Supercomputing Applications at the University of Illinois at Urbana-Champaign.

\section{REFERENCES}

[1] R. F. Harrington, Field Computation by Moment Methods. New York: MacMillan; Florida: Krieger Publishing, 1983.

[2] T. Itoh and R. Mittra, "Spectral domain approach for calculating the dispersion characteristics of microstrip line," IEEE Trans. Microwave Theory Tech., vol. MTT-21, pp. 496-499, 1973.

[3] T. Itoh, "Spectral domain immitance approach for dispersion characteristics of generalized printed transmission lines," IEEE Trans. Microwave Theory Tech., vol. MTT-28, pp. 733-736, July 1980.

[4] Y. L. Chow, J. J. Yang, D. H. Fang and G. E. Howard, "Closed-form spatial Green's function for the thick substrate," IEEE Trans. Microwave Theory Tech., vol. 39, pp. 588-592, Mar. 1991.

[5] _Modeling of electromagnetic fields in layered media by the simulated image technique," in Directions in Electromagnetic Wave Modeling, H. L. Bertoni, and L. B. Felsen, Eds. New York: Plenum, 1991.

[6] M. I Aksun and R. Mittra, "Derivation of closed-form Green's functions for a general microstrip geometry," IEEE Trans. Microwave Theory Tech., vol. 40, no. 11, pp. 2055-2062, Nov. 1992.

[7] T. K. Sarkar, "A note on the choice weighting functions in the method of moments," IEEE Trans. Antennas Propagat., vol. AP-33, pp. 436-441, Apr. 1985.

[8] T. K. Sarkar, A. R. Djordjevic, and E. Arvas, "On the choice of expansion and weighting functions in the numerical solution of operator equations," IEEE Trans. Antennas Propagat., vol. AP-33, pp. 988-996, Sept. 1985.

[9] K. J. Webb, P. W. Grounds, and R. Mittra, "Convergence in the spectral domain formulation of waveguide and scattering problems," IEEE Trans. Antennas Propagat., vol. 38, pp. 869-877, June 1990.

[10] H. Massoudi, C. H. Durney, and M. F. Iskander, "Limitations of the cubical block model of man in calculating SAR distributions," IEEE Trans. Microwave Theory Tech., vol. 32, pp. 746-752, Aug. 1984.

[11] M. J. Hagmann, "Comments on 'Limitations of the cubical block model of man in calculating SAR distributions'," IEEE Trans. Microwave Theory Tech., vol. MTT-33, pp. 347-350, Apr. 1985.

[12] N. Jaochimowicz and C. Pichot, "Comparison of three integral formulations for the 2-D TE scattering problem," IEEE Trans. Microwave Theory Tech., vol. 38, pp. 178-185, Feb. 1990.

[13] R. A. York, R. C. Compton, and B. J. Rubin, "Experimental verification of the 2-Drofftop approach for modeling microstrip patch antennas," IEEE Trans. Antennas Propagat., vol. 39, pp. 690-694, May 1991.

[14] S. L. Sobolev, Partial Differential Equations of Mathematical Physics. New York: Dover, 1989.

[15] O. D. Kellogg, Foundations of Potential Theory. New York: Dover, 1953.

[16] J. G. Fikioris, "Electromagnetic field inside a current-carrying regions," J. Math. Phys., vol. 6, pp. 1617-1620, Nov, 1965.

[17] T. M. Apostol, Mathematical Analysis. Reading, MA: Addison-Wesley, 1974.

[18] J. R. Mosig and F. E. Gardiol, "General integral equation formulation for microstrip antennas and scatterers," Proc. Inst. Elec. Eng., pt. H, vol. 132 , pp. $424-432$, Dec. 1985.

[19] M. I. Aksun and R. Mittra, "Estimation of spurious radiation from microstrip etches using closed-form Green's functions," IEEE Trans. Microwave Theory Tech., vol. 40, no. 11, pp. 2063-2069, Nov. 1992.

[20] M. I. Aksun and R. Mittra, "Spurious radiation from electronic packages," submitted for publication. 
M. I. Aksun (M'92) received the B.Sc. and M.Sc. degrees in electrical and electronics engineering from the Middle East Technical University, Ankara Turkey, in 1981 and 1983, respectively, and the Ph.D. degree in electrical and computer engineering from the University of Illinois at Urbana-Champaign in 1990 .

From 1990 to 1992, he was a Post Doctoral Fellow at the Electromagnetic Communication Laboratory, University of Illinois at Urbana-Champaign. Currently, he is an Assistant Professor at the Department of Electrical and Electronics Engineering, Bilkent University, Ankara, Turkey. Dr. Aksun's research interests include the numerical methods for electromagnetics, $\mathrm{mi}$ crostrip antennas and microwave and millimeter-wave integrated circuits.
Raj Mittra (S'54-M'57-SM'69-F'71) is the Director of the Electromagnetic Communication Laboratory of the Electrical and Computer Engineering Department and Research Professor of the Coordinated Science Laboratory at the University of Illinois. He is a Past-President of AP-S, and he has served as the editor of the Transactions of the Antennas and Propagation Society. He won the Guggenheim Fellowship Award in 1965 and the IEEE Centennial Medal in 1984. He has been a Visiting Professor at Oxford University, Oxford, England and at the Technical University of Denmark, Lyngby, Denmark. Currently, he serves as the North American editor of the journal AEÜ. He is President of RM Associates, which is a consulting organization providing services to several industrial and governmental organizations.

His professional interests include the areas of computational electromagnetics, electromagnetic modeling of electronic packaging, radar scattering, satellite antennas, microwave and millimeter wave integrated circuits, frequency selective surfaces, EMP and EMC analysis, and remote sensing. 